\title{
Research on Web Development Paradigm based on MVC Pattern and Agile Development Mode
}

\author{
Xinhua Zhang ${ }^{1,2}$, Zhiyan Zhang $^{2}$ \\ ${ }^{2}$ Department of software, Anyang normal university, Anyang, Henan, 455000, China; \\ ${ }^{1}$ Department of Computer, Wuhan Vocational College of Software and Engineering, Wuhan, Hubei, \\ 430205, China.
}

Keywords: Web Development, MVC Pattern, Agile Development, Mode, Optimization.

\begin{abstract}
In this paper, we conduct research on web development paradigm based on MVC pattern and agile development mode. At present, the technology of the development of web sites have a lot, from the initial CGI, now to the widespread use of ASP, PHP, JSP, ASP.NET, etc., each technology has its advantages and disadvantages and in the development of different sites, need according to the website functional requirements, facing audience and the special factors such as traffic, developers, choose a different technology. Under tis basis, this paper proposes the MVC pattern and the agile development based paradigm that will enhance the performance of the web design.
\end{abstract}

\section{Introduction}

Website design must be fully attract the attention of visitors, let visitors to produce the enjoyment on the vision. So when I was in a web page authoring the overall site design must be closely combined with the related principle of web design. Network as a new type of the modern society information dissemination media, since the last century 60s was born with extraordinary speed development, into every corner of society, is closely related to people's life. Network is with the aid of network protocol built includes the hardware, software and data resources comprehensive application system [1-2].

According to the survey, we could summarize the techniques for web development as follows. (1) CGI technology. CGI is the meaning of common gateway interface. CGI is a program that is HTTP server program to "talk" with other machines of a tool, the procedure shall be run on a web server, and provide the client interface of HTML pages. (2) ASP technology. ASP is a development environment on the Web server, it can be used to produce and implement dynamic, interactive, high-performance Web service applications and ASP using scripting language as a development language. (3) The PHP technology. PHP is the abbreviation of English super text pretreatment. PHP is a HTML embedded type of language is a server-side implementation of embedded scripting language as HTML document language style is similar to the $C$ language that is now a lot of web programming is widely used. (4) JSP technology. JSP technology using the Java programming language class XML tags and Scriptlets, processing logic to encapsulate generate dynamic web pages. Page also can be accessed through the tags and Scriptlets exist in the server-side resource application logic. JSP page logic and the Web page design and display of separation, support reusable component-based design, make the development of a web-based application quickly and easily.

\section{Our Proposed Methodology}

The MVC Pattern. The MVC design pattern to generate organized modular application as is proven effective method. The MVC design pattern can be the application's data, views, and separate control, enables the presentation layer to separate from the logic business layer, realize the application of loose coupling, so as to improve the efficiency of application development, reliability, maintainability and the extensibility. MVC can be lots of web application system as the main framework, the main reason is that it has the advantages of large and numerous this is mainly embodied in the following several aspects. (1) Because of MVC is a kind of thought is a potential framework structure, so we can on the basis of this model is set up customized application framework, 
in order to meet personalized needs of customers. (2) Between the view and controller, is transparent and independent of each other. This kind of characteristic, allows developers dynamically according to need custom relationship between the controller and view, in order to develop relatively complicated application system. (3) MVC can make a working model, while at the same time establish and use multiple views, and have a special mechanism in MVC and its built-in data monitoring mechanism, depend on the observer pattern.

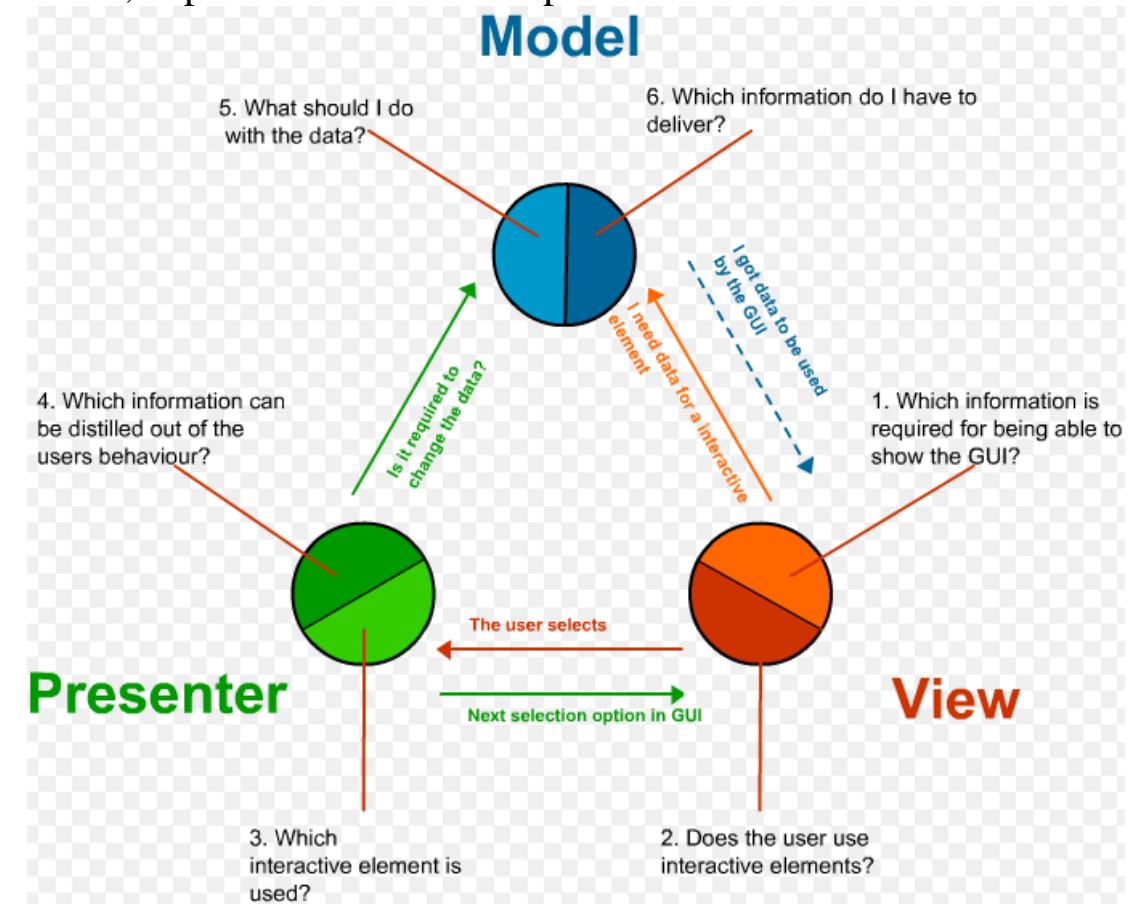

Fig. 1 The Architecture of the MVC Design Pattern

General speaking, the design patterns are abstract summarizing some experience in the design of object-oriented software as it is applicable to the whole field of software development, not for one kind of the specific development. As a result, these experiences can guide the development of Web applications, realize flexible system architecture, changes in demand, adjust the system conveniently, and with minimum cost makes the original system to adapt to the new requirements [3].

Agile Development Mode. Agile development is a human-centered, the iterative and incremental development method, compared with the traditional software development method of "agile", more emphasis on the programmer team work closely with business experts, face to face communication, frequent delivery of new software version, compact and self-organizing team as is able to adapt to the demand change of coding and team organization method, people also pay more attention to software development. Agile development configurable software requirements of the system while the system configuration is usually done by some of the basic parameters setting, when too many parameters are configurable which will be very complex, bring the difficulties to manage, but also may affect system performance. In addition to the parameters configuration, agile development platform as also supports by creating a JSP code file system configuration that can be summarized as follows.

o Maintainable development and the new project development and there are so many different, maintenance and development of prototype, which limited the scope of development that also provides the certain model, which can make full use of the original development interface, do adjustment in the original development interface with fully communication with the needs of the business department.

o The internal encoding specification refers to the development of coding is must abide by the development team to develop or specification approved by agreement. The internal encoding standard is very important, especially for agile development methods [4].

o Developers refer to is responsible for implementing the general requirements of the business requirements. Mainly be responsible for the complete system development work, including requirements analysis, modeling, system design, coding, testing, etc. Requires strong demand 
analysis and processing ability, we must have the strong ability of technology to be able to in the actual development on demand content has overall control and processing capacity.

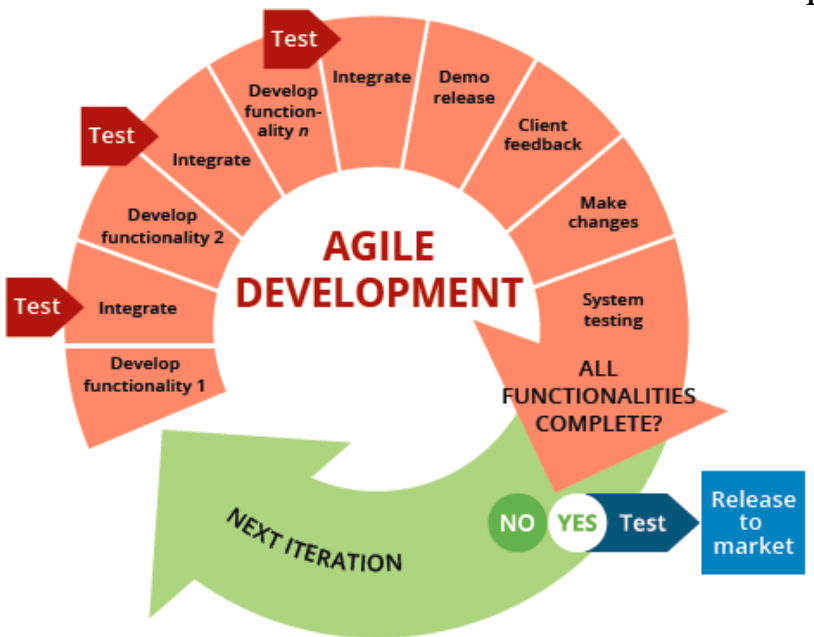

Fig. 2 The Steps of the Agile Development Mode

Website Optimization. Search engine optimization (SEO) by understanding how search engines crawl the Internet page, indexing and that determine for a particular keyword search results ranking technology, such as the optimization of general related web pages for, make its improve search engine rankings, thereby increasing site visits, eventually improve website sales ability or technical ability.

When a website not only consider the site support platform, the structure of the site Settings, page layout, the functions of applicability and background operation simple, more to when writing code to reasonably website optimization element to improve weight of deployment and distribution. Under this background, we should optimize the web as the follows. (1) The title of each page should be with novelty and not to have exactly the same as each into a hierarchy, the content on this page on the front, followed by the name of the website, plus some in the middle of the path. (2) Site map can convenient search engine "spider" crawled page provide the link of your web site, as a kind of potential landing page can be optimized for search traffic, increasing important content. (3) Don't put the script code and style file embedded in a web page code, and try to reduce number of the script file, so conducive to web page loading speed, can use Baidu SEO suggestion tool to inspect, also is advantageous to the search engine crawl. (4) Do not use flash, such as images, Javascript display and load the important content, or links. Search engine does not currently recognize and grab the content in the text, they both influence web page open speed, more conducive to included [5].

The layout of the forms available related research to improve the usability of the visual process. Form elements corresponding labels instead of vertical alignment can improve readability to reduce the operation time, and increase the efficiency of the form, as far as possible using a drop-down box instead of input box, and after the page fault can save the input information avoid duplication of input.

Website Visitor Volume. Website traffic analysis system is as the analyzer arises at the historic moment under this trend as is to provide web site page access count, ranking, and access to analysis services system, it can access to the entire site and any page flow according to the need for a variety of technical analysis, and provide a full comprehensive report to the site manager, make them on their web site conditions have a clear understanding of overall operation. Based on port traffic monitoring real-time traffic monitoring management and performance monitoring module that is the core of the system module as on the basis of traffic acquisition module, we can through the form of chart intuitive selected port traffic situation with the real time monitoring port flow conditions. Monitoring includes: real-time traffic monitoring, port traffic monitoring.

Usually, the flow analysis system should be able to give at least the following aspects of statistical data. (1) Page: including website sort of visits each directory; Site visits of each file number. (2) The visitors to the region statistics: including classification, according to the source IP address and press the access frequency sorting as based on IP areas classification according to the province, country, it 
is concluded that the region of total traffic. (3) Total flow statistics: including website traffic statistics and time span as website traffic time grouping sorting, which time can choose in hours, days, weeks, etc. (4) According to the special needs of the managers choose other additional information, such as visitors use the type of browser and operating system and so on.

Web Development Paradigm. Appeared with the development of the network, cable network and basic wireless network two network patterns, the emergence of the network model, makes the people in your life for the application of network is more convenient and quick, the dependence of the people for network is becoming more and more strong, and people at the same time, a large number of the applications in the network for network application requirements are constantly ascension.

Website development in the construction of database of the main is to meet customer demand as the focus, in the process of building the database, need according to the functionality of the site and size to planning in the form of database building. On database design, need before the design from the points of the demand of market to conduct a comprehensive, to plan out the concrete implementation plan, the abnormal problems may arise in the process of building a reasonable modification, and thus makes the website of the late maintenance more convenient. Thus, the advantages and disadvantages of each technique, they are respectively applied to the different needs of website development, grasp the different technical personnel in website development technology that will have a different choice. Through the above comparative analysis, expectations for the new site team in the development of the technology of choice to provide some valuable reference.

\section{Final Conclusion}

In this paper, we conduct research on web development paradigm based on MVC pattern and agile development mode. The Internet has become the main channel to spread information, provide service and communication, thus improve website accessibility is particularly important and urgent. From the government, the education sector to enterprises and institutions have site should consider adopting website accessibility design guideline recommendations. With this goal we propose the new paradigm for the web design, in the future, we will implement the techniques with projects.

\section{Acknowledgement}

This paper is supported by: 1 . Vocational education in the Hubei province to learn subject: the IOT professional practice reform based on Scrum (ZJGB201515). 2. Wuhan city bureau of education subject: IOT professional practice teaching reform based on agile development (2015118). 3. Hubei province education science "twelfth five-year" plan: Research on the development of the software technique major of general higher vocational teaching resource construction in ubiquitous learning environment (2015GA076).

[foundation item. Hubei Provincial Vocational Education Society topic "based on scrum networking professional practice reform" ZJGB201515; 2. Wuhan City Bureau of education subject "based on agile development of networking professional practice teaching reform" (2015118); 3. Hubei Province Education Science "1025" planning "ubiquitous learning environment software technology specialty in higher vocational education development course teaching resources storehouse construction of 2015GA076)

\section{References}

[1] Fernandez, Adrian, Silvia Abrahão, and Emilio Insfran. "Empirical validation of a usability inspection method for model-driven Web development." Journal of Systems and Software 86.1 (2013): 161-186.

[2] Park, Thomas H., Brian Dorn, and Andrea Forte. "An analysis of HTML and CSS syntax errors in a Web development course." ACM Transactions on Computing Education (TOCE) 15.1 (2015): 4. 
[3] Chao, Joseph, Kevin Parker, and Bill Davey. "Navigating the framework jungle for teaching web application development." Issues in Informing Science and Information Technology 10 (2013).

[4] Johnson, Larry, and Annette Lamb. "Riding the Winds of Change: New Directions for Libraries and Web Development Tools." (2013).

[5] Gupta, Pranshu, Ramon Mata-Toledo, and Morgan Monger. "Utilizing ASP. NET MVC in web development courses." Journal of Computing Sciences in Colleges 27.3 (2012): 10-14. 